\title{
Learn Smarter, Not Harder - Exploring the Development of Learning An- alytics Use Cases to Create Tailor-Made Online Learning Experiences
}

\author{
Eva Ritz \\ University of St. Gallen \\ eva.ritz@unisg.ch
}

\author{
Timo Grüneke \\ FIM Research Center, University of Bayreuth \\ Project Group Business and Information Systems \\ Engineering of Fraunhofer FIT \\ timo.grueneke@,fit.fraunhofer.de
}

\begin{abstract}
Our world is significantly shaped by digitalization, fostering new opportunities for technology-mediated learning. Therefore, massive amounts of knowledge become available online. However, concurrently these formats entail less interaction and guidance from lecturers. Thus, learners need to be supported by intelligent learning tools that provide suitable knowledge in a tailored way. In this context, the use of learning analytics in its multifaceted forms is essential. Existing literature shows a proliferation of learning analytics use cases without a systematic structure. Based on a structured literature review of 42 papers we organized existing literature contributions systematically and derived four use cases: learning dashboards, individualized content, tutoring systems, and adaptable learning process based on personality. Our use cases will serve as a basis for a targeted scientific discourse and are valuable orientation for the development of future learning analytics use cases to give rise to the new form of Learning Experience Platforms.
\end{abstract}

\section{Introduction}

The education industry is currently experiencing a blurring of boundaries from traditional face-to-face to online-centric learning environments [1]. Not only due to the recent COVID-19 pandemic but also through recent progress in digitalization, this change has been accelerated [2]. Thus, digital education platforms like Coursera, Udemy, Edx, or Udacity are conquering markets worldwide, offering online courses on manifold subjects. There is much educational information available worldwide, promoting new possibilities for technology-mediated learning. Especially organizations need to develop dynamic capabilities [3] and must pass on essential knowledge for digital transformation and innovation to their employees [4].

However, during the learning process, individuals and organizations get overwhelmed through an information overload caused by news, websites, search engine results, and other online sources [5]. Consequently, digital education platforms should respond by offering individualized learning experiences, which are tailor-made for every user [6]. Individualized learning experiences promise, amongst others, to increase learning outcomes [7], improve learning effectiveness [8], stimulate intrinsic activation, and motivation of learners [6].

In addition to typical e-learning platforms and Learning Management Systems (LMS), learning analytics (LA) currently gains ground as it creates a novel and innovative learning experience. While the availability of online learning data from these platforms expands rapidly, the application of LA methods enables individualized education. The goal of LA is to derive direct interventions and actions for the learner to improve the learning behavior and progress [9], and to ensure optimal receptivity of the learner [10].

Through individualized learning experiences, users can access relevant knowledge that is tailored to their personality, knowledge state, or preferences [11]. Besides, attention must be paid to the optimal didactic design of individualized learning experiences. Different learning personalities should be addressed for optimal absorption, processing, and consolidation of content [12]. A holistic personalized learning experience has a positive impact on learner motivation and success [13].

Despite a broad body of literature dealing with digital education platforms, the scholarly discourse has not yet succeeded in taking a holistic view of learning experiences and in linking and combining different LA use cases. Due to the high relevance of learning smarter and individualized in combination with the 
still missing holistic view to individualize learning experiences through LA, we pose the following research question: How can learning analytics facilitate individualized learning experiences on digital education platforms?

To answer this research question, we defined LA use cases concerning individualized learning experiences and conducted a systematic literature review of individual LA applications. Hence, we merged research papers in a structured analysis to provide a systematic overview of the use case and discuss the technical application. With our research, we want to shed light on the various possibilities of individualization during the learning processes in a systematic way. Further, the use cases support educators and educational institutions to foster the technical implementation of LA in a holistic technology-mediated environment.

\section{Theoretical Background}

\subsection{Technology-Mediated Learning Platforms}

In the past, technology-mediated learning platforms have evolved from mere content delivery to highly advanced technological platforms [14, 15]. A platform is an "extensible codebase of a softwarebased system that provides core functionality" [16]. Educational platforms for content delivery are also more commonly known as e-learning or LMS. They have different functionalities (e.g., content or performance management) that enable a particular learning process to be planned, executed, and fundamentally evaluated. Educators become able to create and digitally deliver content, manage learners, and evaluate their performance [17]. Having already outlined that the development of new organizational capabilities is people-driven, measures need to be taken to ensure knowledge transfer $[18,19]$.

However, digitization is causing the amount of information to grow exponentially. Accordingly, traditional training approaches, such as classroom training, are becoming inefficient. In classroom training, the amount of information cannot be conveyed and is not tailored to the individual's level of knowledge. This is where LA comes in and allows knowledge levels and learner's preferences to be recorded, managed, and automatically used by an intelligent system.

\subsection{Learning Analytics}

LA involves the use of collected information about learners and learning environments in use to assess, determine, and analyze it for modeling, predicting, and optimizing learning processes [20]. Here, the focus is on measuring and analyzing learner data. This includes, for example, learning interaction data on the use of the education platform, learning activities, or learning assessment results [21]. The overall goal of LA is to optimize learning and teaching based on intelligent data analysis. From a pedagogical perspective, the intelligent learning system should interact like a personal teacher, helping to improve the user's learning outcome [22]. In this regard, the implementation of LA is not only important in the academic field but also organizational training. In this context, continuous development of employees' capabilities is a key success factor for the organization [4].

To further describe the process of applying LA, Clow [23] defined a LA cycle in four steps: (1) the learner generates data, (2) the data about the learner and the learning behavior is collected, (3) the data is analyzed and visualized, and (4) interventions are derived on this basis to improve the learner's learning behavior. This last step is an essential component, as the data collected should always trigger interventions in favor of the used learner's data, so that the learning outcome is improved [24]. For this reason, the learning environment should provide real-time feedback to the learner to close the cycle [22].

However, the goals and methods of LA should be considered more closely to identify and implement appropriate use cases [25]. The reference model for LA by Chatti et al. [25] identifies four dimensions for shaping use cases: The data environment, the stakeholders of the data environment, the goals, and the methods. The goals and methods of LA should be considered more closely to identify and implement appropriate use cases [25]. The goals can trigger different interventions in learners and thus contribute to different purposes in the learning process. In this context, Seufert et al. [26] distinguish at the individual learner level between analytics use cases for reflection and for prediction. The former refers to the evaluation and visualization of the learning outcome in the form of feedback after the learning process. This includes, for example, learning level reviews or evaluations of the learner. Second, predictions aim to generate a predictive and adaptive learning system, which includes recommendations and personalization. LA methods are mostly based on the implementation of data science or artificial intelligence (AI) applications. Chatti et al. [25] include statistical methods, visualizations, classifications and predictions, clustering, data mining, or social network analysis. 


\section{Research Method}

To answer our research question and to give a comprehensive overview of how LA facilitates individualized learning experiences, we defined literaturebased use cases, collected data from research papers, and systematically analyzed them. By drawing from a selection of 42 papers we derived four use cases. Thus, we were able to engage in explanation building (type two) for the field of information systems [27].

The definition of literature-based use cases aims to identify and analyze the use of LA for individualized learning experiences on educational platforms. In the first step, we conducted an initial literature screening to become acquainted with the established LA methods and identified promising use case areas. To answer our research question, we applied the following criteria for LA use cases to be included in our selection: (1) use case must have a clear objective and appropriate LA methods, (2) use case must focus on individual LA systems (no social LA systems), (3) use case allows to create direct value for the learner. After the initial screening, we conducted brainstorming sessions and identified patterns, based on which we finalized the use case patterns to ensure that the sampling is purposive, logical, and representative [28]. The result is the following four use case patterns: learning dashboards (n1), individualized learning content (n2), individualized tutoring (n3), and personalized learning systems (n4).

In the second step, we follow Kitchenham and Charters [29] to execute a structured literature search for the data collection of our use cases. For each use case, brief definitions are drawn up for the purpose of subdivision, and together with our research question, the following search terms are derived from these definitions: (n1)= "learning AND dashboard", (n2) = "individualized AND learning AND (path OR content)", (n3)= "individualized AND tutoring", (n4) = "adaptive AND learning AND personality". By applying our search strings, we initially found $(\mathrm{n} 1=249 ; \mathrm{n} 2=70 ; \mathrm{n} 3=49 ; \mathrm{n} 4=34$; in total $=402)$ relevant articles using the IEEE Xplore Digital Library. By focusing our search only on IEEE Xplore, we were able to incorporate the technical engineering of LA use cases and address our interdisciplinary research field. The literature search in all metadata (conducted in May 2021) includes journal articles and conferences as of 2010 to guarantee up-to-date research.

For our identified articles we adopted inclusion criteria to narrow down our set of articles to $(n 1=8$; $\mathrm{n} 2=10 ; \mathrm{n} 3=15 ; \mathrm{n} 4=9$; in total $=42$ ). To conduct this selection, all authors screened titles and abstracts and scored the articles for relevance to the use case. The scoring ranged from "low" ( to the use case) to "high" ( score $=4$; the article has a clear connection to the use case). Only articles that scored four were included in our final selection which adds up to 47 articles. During this process, we removed five duplicates appearing in two use cases and assigned them to the more suitable use case resulting in our total of 42 articles.

In the third step, we applied selective coding. In line with Wolfswinkel et al. [30], this is done by assigning text excerpts to categories. Based on the LA framework of Seufert et al. [26], we used their four dimensions for the analysis: objectives, data sources, interventions, and applications. We adopted these dimensions as categories to analyze the use cases and ensure comparability during the analysis.

The dimension objectives aims to determine how LA can support reflection or prediction in the learning process [26]. Data sources consider data requirements of the learner e.g., age, gender, personality, or system interaction. As the intervention for the learner is the most important factor [23], we included it as a separate dimension. Interventions propose the pedagogical actions on the learners to improve their learning outcomes. The fourth dimension comprises the application, which includes the used methods, technologies, and technical implementation in the education platform. In contrast to Seufert et al. [26] and Greller and Drachsler [7], we excluded the stakeholder dimension because our research question explicitly aims to identify use cases for the learner only.

\section{Results}

\subsection{Use Case 1: Individual Learning Dash- board}

Individual LA dashboards are often implemented on digital education platforms to track learners' skill development and learning activities, as well as to detect an emerging problem during the learning process [31]. Learning dashboards often comprise a singlepage user interface to display the current learning state and historical key performance indicators [32, 33]. The visualization should facilitate self-monitoring learning activities, enable informed decisions, and influence the intended learning behavior of the individual e.g., by evaluating the performance. Thus, the visualization attempts to influence the learner's psychology and activities to learn more effectively [34].

Objectives. Learning dashboards generally aim to evoke changes in learning behavior through visualization [35]. In the context of sense-making, LA dashboards aim to represent user-specific learning data in a meaningful way $[32,35]$. Besides, dashboards can provide personalized, visualized feedback for learners 
in real-time [36, 35]. This visually-prepared feedback also provides data-driven insights to improve the decision-making processes of learners, for instance about the learning process and progress [32, 36, 31, 35]. Schwendimann et al., Matcha et al., and Rohloff et al. $[32,31,35]$ have experienced that LA dashboards can improve the motivation and behavior of learners. In line with the constructivist learning approach, a major objective of dashboards is the facilitation of self-monitoring, self-regulation, and self-evaluation [32, 35].

Data sources. The most important data input referring to Schwendimann et al. and Matcha et al. [32, $31]$ is logs to track computer-mediated user activity. However, there are manifold data sources that can be used for the application of LA dashboards, for instance, user information collected through questionnaires and interviews [32, 31], user-produced learning artifacts, completed tasks [32, 33], and test scores [36, 33], institutional database records including course information [32], or user activity tracked with sensors [32]. Additionally, Schwendimann et al. [32] examine the advantages of collecting data from external application programming interfaces (API) and social media applications.

Interventions. Dashboard-induced interventions mainly focus on evoking a change in learning behavior [35]. The interventions of analyzed use cases induce, amongst others, the adaption of learning strategies and the update of goals and standards. Further, interventions might influence learner's motivation, self-efficacy, and dispositions [31]. According to Schwendimann et al. [32], interventions base on one or more of the following dimensions: the learners' description, their actions on the platform, the produced and learned content, their results, the learning context, and their social interaction. Rohloff et al. [35] differentiate between progress and performance interventions. While progress overviews provide information on which learning material must still be completed during the learning process, performance overviews can induce repetition suggestions after assessments. Matcha et al. and Rohloff et al. [31, 35] state an example for social comparison, an often-applied intervention where dashboards show the results of the individual compared to the average class. The visualization of an individualized learner's activity compared to cohorts stimulates self-reflection.

Application. For the technical implementation of dashboards, three components must be integrated. A useful dashboard should combine functionality and interactivity for the users [37]. Therefore, usability testing is an important step during dashboard development and implementation [37]. The first components are web-based learning environments or LMS, for in- stance, moodle or Edx [36, 33, 35]. The second component is a visual representation tool implemented in the graphical user interface. Proper visualization tools include most commonly bar charts, line graphs, tables, timelines, circular progress bars, pie charts, histograms, network graphs, or boxplots $[32,36,35]$. For instance, Ramaswami [36] facilitates the deployment through Power BI services. Concerning the dashboard, not only a single-page overview but also the integration of smaller widgets during the learning process are useful [35]. The third component is the application of statistical methods to calculate key performance indicators and other performance scores. This includes the use of Machine Learning algorithms for pattern extraction and classification e.g., from historical use engagement and learning behavior of previous student cohorts [36]. In the same context, Volaric and Ljubic [33] propose a fuzzy analytic hierarchy process to evaluate trace attributes of learners during testing and assess their domain knowledge.

\subsection{Use case 2: Individualized Learning Con- tent}

A second important part of tailor-made learning experiences is the individualization of content, learning paths and processes. Individualized learning content and learning paths enable learners to consume customized knowledge. Based on the learner's past learning data and the data of similar users, a recommender system can recommend future content [38]. Content individualization is often accompanied by a microlearning approach, which is the accumulation of multiple small learning sequences [39].

Objectives. By identifying personal user preferences and domain knowledge, LA applications aim to individualize knowledge content [5, 8, 40-44]. Matching the recommended content to the learner's prior knowledge can improve learning effectiveness[43, 8].

Data Sources. Because we require information about the learner's preferences, data sources include user learning data from the LMS, integrated development environments, and users' profile information [8]. Brinton et al. [44] also recommend including data on the learner's browsing behavior and clickstreams. Furthermore, to assess the knowledge state of the learner, the user's data on prior knowledge, test data, and the learner's historical log information must be included [45].

Interventions. To increase the learners' enthusiasm and improve the learning effect, LA applications suggest a personalized learning path to the user [40, 45]. Raman and Nedungadi [43] mention that the adaption can comprise the format of learning content, the order of content, and the navigation flow of the 
user. Brinton et al. [44] add that learning paths can be adapted for improvements based on user feedback. Moreover, the prediction of a user's real-time knowledge state enables the insertion of recommendation nudges for further content [40]. A different intervention is the suggestion of interactive course materials based on a user's clickstream data [44].

Application. The use case includes the generation of individualized learning paths based on learning behavior [45, 46] or learning content $[40,43,5]$. In general, some authors execute the individualization of learning paths on the web environment, whereas others focus on describing the algorithms used for the individualization. For instance, Kardan and Speily [40] ground their individualized content on an open LMS that can include and update suitable learning contents from web sources (use filtering algorithms to include only relevant and up-to-date sources). Hence, $\mathrm{Na}$ et al. and Samia and Abdelkrim [46, 42] use an adaptive elearning hypermedia system for tailoring content to a learner's objectives, learning type, knowledge state, and personal interests. Commonly used LA methods for the assimilation of learning content include clustering, recommendation, and optimization algorithms. These different methods are not exclusive, but can also be combined in the use cases [44]. One approach of clustering is an analysis based on a learning behavior similarity calculation method (e.g., Kull-back-Leibler divergence matrix) with comparable learning types [45]. Recommender systems calculate content similar to user's preferences and then recommend an appropriate content list $[42,5,44]$. To state an example, $\mathrm{Na}$ et al. [42] apply a recommendation algorithm based on knowledge representation. Besides, Huang and Lu [5] compare typical recommender systems like collaborative filtering, content-based, or knowledge-based recommendation algorithms for the individualization of the learning path. For the application of optimization methods, Govindarajan [8] uses a parallel particle swarm optimization mechanism for the analysis and prediction of adapted learning paths based on proficiency.

\subsection{Use case 3: Individualized Tutoring}

A key aspect of learning is engaging learners to stimulate high attention and motivation. In traditional classroom settings, instructors can interact with learners and tutor them one-on-one, if needed. This is not possible in digital education, especially in environments where content is provided on-demand. Thus, instructors or learning facilitators in the past had to consider and map every possible path through the learning content to anticipate optimal learning paths [47]. To- day AI applications (or humanoid robots [48]) facilitate this process automatically and adapt to the learner's need [47] based on data traces [49]. We call these applications intelligent tutoring systems (ITS) designed to individualize learning according to the learner's needs and learning styles [48, 49].

Objectives. Through dynamical motivation, ITS allow the learner to improve their learning experience [50]. This is enabled by monitoring learner engagement and deploying strategies to maintain attention [48]. This monitoring occurs via the collection and analysis of behavioral data that further complements the learner model [51]. Preferably, the system is selflearning and adapts incrementally to new data [52]. This allows the ITS to give direct feedback and aid the learner $[51,53]$. For instance, this can be achieved by providing the learner with hints about a particular exercise they are working on [47]. Nonetheless, one central component should not be neglected: emotions. When considered, this changes an ITS to an affective tutoring system (ATS). During learning, the emotional state of the learner is captured through appropriate emotion recognition channels so that the ATS can resolve any obstacles [54].

Data sources. As we investigate the general structure of an ITS or ATS, the learner model represents an important part to successfully capture the details of the learner to attain the objective of individualized tutoring [55]. Thus, it is the aim to capture data and model this raw data [56]. To ensure intertemporal comparisons, time data should be tracked [56]. Learners' actions are useful [56] and comprise interactions between learner and content [51]. The learning data together with historical data [51], and administrative data [47] can then be processed. Additionally, learning styles of the learner [57], dynamic learning preferences, motivation states, learners preferences, and capabilities [53] are insightful for the intervention later on. Moreover, self-assessment quiz-questions and confidence in their answer [58] provide insights into pre-existing knowledge $[53,47,51,57]$. Data on the frequency of use and duration of use or question answering and task difficulty are also informative [47].

Interventions. Based on the learner model executive systems initiate action to guide the learner [47]. The feedback contains explicit information on what mistakes have been made or how they could have been avoided [59]. A variety of measures are applicable. Instructions that explain step-by-step the wrong-doing, or complementary tutorials based on the learning style [59]. Also, individualized hints or nudges are interventions that can guide the learner $[57,59,50]$. It may also provide recommendations for group collaboration based on the data [57]. If the system cannot provide 
sufficient service in-person tutoring sessions can be initiated [60]. But also positive incentives through trophies or badges pose an intervention to keep learners engaged [57, 50]. Furthermore, physical communication via humanoid robots is possible to instruct and motivate learners [54].

Application. Analysis of the data is necessary to implement the appropriate interventions. Lian [53] achieved this goal through a simple hierarchical model in combination with a Bayesian student model and rule-based models for actions based on Kolb's theory. Further, the Bayesian knowledge tracing model can access learner's knowledge and knowledge gain too but also incorporates the decay of knowledge over time [61]. The random forest classifier can estimate the knowledge of learners at a low cost of training and is relatively stable [58]. Also, deep adaptive resonance theory networks combined with development learning networks can handle the learner status, learning preference, and the learner experience [52]. Acting on data planning and sequencing with the help of acyclic graphs is feasible to accomplish an instructional goal [47]. An interesting application is the use of natural language processing as it allows to catch and evaluate errors (e.g., grammatical or careless errors) made in text-based tests [50]. A more complex AI application is the prediction of user behavior through case-based reasoning. Here, each learner can be seen as a case that needs to reach a target case state that has been solved in the past [56]. Hence, case-based reasoning is a suitable approach and aims to use past experience to solve the new case [49]. In this regard, the method is more successful if a sequence in the learning behavior is as similar as possible when comparing the current use case with past use cases, and can be done using the inverse longest common subsequence measure [56].

\subsection{Use case 4: Adaptive Learning Based on Personality}

An adaptation of the learning environment can also support the learners. This is mainly about adapting the system to personal learning characteristics. In research, different models are applied to distinct personal characteristics. Felder and Soloman [62] developed the VAK model to highlight visual, auditory, and kinesthetic learning types. Kolb [63] distinguishes between anticipatory (acting and feeling), divergent (feeling and observing), convergent (acting and thinking), and assimilative (observing and thinking) learning styles. Associations always exist between personality types, learning types, and learning content in online learning [64]. This means, for example, that extroverts prefer learning through discussion and social interaction. Accordingly, the personality of the learner is often applied as a factor to individualize the learning environment.

Objectives. This use case aims to tailor the user experience and user interface to individual characteristics of the learners to accommodate an optimal learning experience adapted to their learning $[65,66]$ and thinking [66] styles. This can be done automatically and manifest through the interaction with the system $[67,11]$. The goal is to gather information about the personality and intelligence traits of the individual to predict at-risk learners' failure [68]. Likewise, we need to understand the relationship between time spent on training and personality to maximize learning time [69].

Data sources. Data points utilized are age, gender, or pre-knowledge [65]. The focus lies on capturing interaction, which comprises attendance, preview, discussion, test, or review [67] as well as time to answer, idle time, times of saving specific answers, and pre-accessed goal expectancy related to questions [69]. In terms of personality traits, the NEO five-factor inventory test (extraversion, neuroticism, conscientiousness, openness to experience, and agreeableness) was used [68]. Likewise, the Big Five Inventory questionnaire for introversion and extraversion [11], extraversion and conscientiousness [69] as well as openness to experience and extraversion [67], are in use. Game scenarios were used to collect the following traces: time, confidence, activities, risk, number of friends, color, gregariousness, and feeling. Tlili et al. [11] use game scenarios to match personality through LA using screen time, confidence, activities, risk-taking, social interactions, and feelings. When we look into learning styles the Felder-Silverman model questionnaire is used (perception, processing, input, understanding) [68]. As for learning personality, Elghali [66] uses his questionnaire for his learner model. Merely Carro and Sanchez-Horreo [68] use a primary mental abilities questionnaire (verbal, spatial, and general reasoning) to apprehend the intelligence of the learner.

Interventions. One possible intervention is the adaption of the content and the sequence of content $[65,46]$. In addition, the recommendation of social activities (individual or collaborative) based on the analyzed learner data is used to adapt the learning experience to the learning personality [68].

Application. Again, the basis is the learner model. One application is fuzzy logic systems to imitate human reasoning and model the learner accordingly [65]. Elghali [66] uses a classification approach with a multilayer perception algorithm and radial basis function network algorithm to identify learning styles [66]. Whereas Lai et al. [67] use an enhanced extended nearest neighbor algorithm for the Big Five Personality (openness to experience and extraversion), Tlili et 
al. [11] use a Naïve Bayes classifier (introversion and extraversion), and Papamitsiou and Economides [69] the method of partial least squares (extraversion and conscientiousness).

\section{Discussion and Conclusion}

After assembling different LA applications, we are confident that learning experiences on educational learning platforms have the prospective to offer individualized learning dashboards, individualized learning processes, intelligent tutoring, and adapt learning environment to personality. Considering the challenge of information overload accompanied by an increasing need for organizational training, tailoring content can improve learning effectiveness [5]. Due to individualized learning, platform users are finally able to study smarter, not harder. Simultaneously, there is also a lasting impact for the organization through savings of time and money, as well as a growing motivation and satisfaction of learners. After conducting our systematic analysis through the application of the criteria of Seufert et al. [26], we observe that not all of the dimensions are included in the use cases identified in the literature. Regarding the four dimensions, often explanations on interventions are missing within the literature base. However, Clow [23] argues that the direct impact on the learner is the most relevant part of LA application. Only if the end-user is directly addressed to improve learning effectiveness and behavior, the value creation process for the organization takes place. We recognized that usability testing can be a valuable approach to ensure and test interventions from technical and pedagogical perspectives (e.g., $[34,37,70]$ ).

While reassessing our results, we concluded that a holistic point of view and implementation of LA approaches on the digital education platform is inevitable. We, therefore, propose the expansion of the traditionally used term "LMS" into "Learning Experience Platform" or LXP, which aims to comprise a fully individualized learning experience for the user. This evolution requires also APIs to enable the inclusion of other data sources and platforms but more importantly interoperable standards (e.g., xAPI and cmi5) to get a complete picture of the learner on the way to a holistic individualized experience [32]. Thus, it also requires methods to tackle the resulting data variety in the technical application of the LXP. In this context, also data security and regulation must be discussed for the widespread implementation of LXPs.

Our research study is not without certain limitations. First, we focused the individualized learning experience use cases only on the learner as a platform stakeholder to analyze direct value creation in their learning processes. However, educational platforms cover many stakeholders (e.g., teachers or instructors, their institutions, and organizations), which are excluded in this study. Second, extending the use case sample could widen the research scope and increase the generalizability (e.g., upcoming topics like the integration of sentimental analysis, virtual and augmented reality, or smart personal assistants in the learning process).

Further research is necessary to engage in the interconnectivity and symbiosis of individualized learning experience use cases to create a systematic and holistic digital LXP to study smarter. We suggest that a beneficial approach of LA applications should combine the detailed technical application aligned with the pedagogical perspective to design useful learning interventions to create tailor-made online learning experiences.

\section{References}

[1] P. Dillenbourg, "The Evolution of Research on Digital Education," Int J Artif Intell Educ, vol. 26, pp. 544-560, 2016.

[2] Top Technology Trends Impacting Higher Education in 2021, Feb, 2021.

[3] D. J. Teece, G. Pisano, and A. Shuen, "Dynamic capabilities and strategic management," Strat. Mgmt. J., vol. 18, pp. 509-533, 1997.

[4] C. Buck, T. Grüneke, and K. Stelzl, "Structuring the Jungle of Capabilities Fostering Digital Innovation," Proceedings of the 16th International Conference on Wirtschaftsinformatik (WI), 2021.

[5] R. Huang and R. Lu, "Research on Contentbased MOOC Recommender Model," in 2018 5th International Conference on Systems and Informatics (ICSAI): IEEE, 2018, pp. 676-681.

[6] D. Nandigam, S. S. Tirumala, and N. Baghaei, "Personalized learning: Current status and potential," in 2014 IEEE Conference on e-Learning, e-Management and e-Services (IC3e): IEEE, 2014, pp. 111-116.

[7] W. Greller and H. Drachsler, "Translating Learning into Numbers: A Generic Framework for Learning Analytics," Journal of Educational Technology \& Society, vol. 15, pp. 42-57, 2012.

[8] K. Govindarajan, V. S. Kumar, and Kinshuk, "Dynamic Learning Path Prediction - A Learning Analytics Solution," in 2016 IEEE Eighth International Conference on Technology for Education (T4E): IEEE, 2016, pp. 188-193.

[9] J. M. Kevan and P. R. Ryan, "Experience API: Flexible, Decentralized and Activity-Centric Data Collection," Tech Know Learn, vol. 21, pp. 143-149, 2016. 
[10] P. Hu, J. Milton, W. Hui, W. Ma, T. Clark, and K. Tam, "Examining e-Learning Effectiveness, Outcomes and Learning Style: A Longitudinal Field Experiment," 9th Pacific Asia Conference on Information Systems: I.T. and Value Creation, PACIS 2005, 2005.

[11] A. Tlili, F. Essalmi, L. J. B. Ayed, M. Jemni, and Kinshuk, "A Smart Educational Game to Model Personality Using Learning Analytics," in 2017 IEEE 17th International Conference on Advanced Learning Technologies (ICALT): IEEE, 2017, pp. 131-135.

[12] A. Moubayed, M. Injadat, A. B. Nassif, H. Lutfiyya, and A. Shami, "E-Learning: Challenges and Research Opportunities Using Machine Learning \& Data Analytics," IEEE Access, vol. 6, pp. 39117-39138, 2018.

[13] B. Gan and C. Zhang, "Research on Design of Personalized Learning Experience Based on Intelligent Internet Technology," in 2020 International Conference on E-Commerce and Internet Technology (ECIT): IEEE, 2020, pp. 306-309.

[14] M. Andergassen, G. Ernst, V. Guerra, F. Modritscher, M. Moser, and G. Neumann, et al., "The evolution of e-learning platforms from content to activity based learning: The case of Learn@WU," in 2015 International Conference on Interactive Collaborative Learning (ICL): IEEE, 2015, pp. 779-784.

[15] S. Gupta and R. P. Bostrom, "Technology-Mediated Learning: A Comprehensive Theoretical Model," Journal of the Association for Information Systems, vol. 10, pp. 686-714, 2009.

[16] A. Tiwana, B. Konsynski, and A. A. Bush, "Research Commentary -Platform Evolution: Coevolution of Platform Architecture, Governance, and Environmental Dynamics," Information Systems Research, vol. 21, pp. 675-687, 2010.

[17] M. Paulsen, "Experiences with Learning Management Systems in 113 European Institutions," Educational Technology \& Society, vol. 6, 2003.

[18] Roberts, Galluch, Dinger, and Grover, "Absorptive Capacity and Information Systems Research: Review, Synthesis, and Directions for Future Research," MIS Quarterly, vol. 36, p. $625,2012$.

[19] J. J. P. Jansen, F. A. J. van den Bosch, and H. W. Volberda, "Managing Potential and Realized Absorptive Capacity: How do Organizational Antecedents Matter?," Acad Manage J, vol. 48, pp. 999-1015, 2005.

[20] D.-K. Mah, "Learning Analytics and Digital Badges: Potential Impact on Student Retention in Higher Education," Tech Know Learn, vol. 21, pp. 285-305, 2016.
[21] International Organization for Standardization, Ed., Information technology for learning, education and training - Learning analytics interoperability - Part 1: Reference model. ISO/IEC TR 20748-1:2016, 2016.

[22] N. Law and L. Liang, "A Multilevel Framework and Method for Learning Analytics Integrated Learning Design," JLA, vol. 7, pp. 98-117, 2020.

[23] D. Clow, "The learning analytics cycle," in Proceedings of the 2nd International Conference on Learning Analytics and Knowledge - LAK'12. S. Dawson, C. Haythornthwaite, Eds. New York, New York, USA: ACM Press, 2012, p. 134.

[24] D. Clow, "An overview of learning analytics," Teaching in Higher Education, vol. 18, pp. 683695, 2013.

[25] M. A. Chatti, A. L. Dyckhoff, U. Schroeder, and H. Thüs, "A reference model for learning analytics," IJTEL, vol. 4, p. 318, 2012.

[26] S. Seufert, C. Meier, M. Soellner, and R. Rietsche, "A Pedagogical Perspective on Big Data and Learning Analytics: A Conceptual Model for Digital Learning Support," Tech Know Learn, vol. 24, pp. 599-619, 2019.

[27] Gregor, "The Nature of Theory in Information Systems," MIS Quarterly, vol. 30, p. 611, 2006.

[28] M. B. Miles and A. M. Huberman, Qualitative data analysis. An expanded sourcebook / Matthew B. Miles, A. Michael Huberman. Thousand Oaks, Calif., London. SAGE, 1994.

[29] B. A. Kitchenham and S. Charters, "Guidelines for performing Systematic Literature reviews," Software Engineering Version 2.3. Engineering, 2007.

[30] J. F. Wolfswinkel, E. Furtmueller, and C. P. M. Wilderom, "Using grounded theory as a method for rigorously reviewing literature," Eur. J. Inf. Syst, vol. 22, pp. 45-55, 2013.

[31] W. Matcha, N. A. Uzir, D. Gasevic, and A. Pardo, "A Systematic Review of Empirical Studies on Learning Analytics Dashboards: A SelfRegulated Learning Perspective," IEEE Trans. Learning Technol., vol. 13, pp. 226-245, 2020.

[32] B. A. Schwendimann, M. J. Rodriguez-Triana, A. Vozniuk, L. P. Prieto, M. S. Boroujeni, and A. Holzer, et al., "Perceiving Learning at a Glance: A Systematic Literature Review of Learning Dashboard Research," IEEE Trans. Learning Technol., vol. 10, pp. 30-41, 2017.

[33] T. Volaric and H. Ljubic, "Learner and course dashboards for intelligent learning management systems," in 2017 25th International Conference 
on Software, Telecommunications and Computer Networks (SoftCOM): IEEE, 2017, pp. 16.

[34] Y. Park and I.-H. Jo, "Development of the Learning Analytics Dashboard to Support Students' Learning Performance," Journal of Universal Computer Science, vol. 21, pp. 110-133, 2015.

[35] T. Rohloff, D. Sauer, and C. Meinel, "Student Perception of a Learner Dashboard in MOOCs to Encourage Self-Regulated Learning," in 2019 IEEE International Conference on Engineering, Technology and Education (TALE): IEEE, 2019, pp. 1-8.

[36] G. S. Ramaswami, T. Susnjak, and A. Mathrani, "Capitalizing on Learning Analytics Dashboard for Maximizing Student Outcomes," in 2019 IEEE Asia-Pacific Conference on Computer Science and Data Engineering (CSDE): IEEE, 2019, pp. 1-6.

[37] R. Bodily and K. Verbert, "Review of Research on Student-Facing Learning Analytics Dashboards and Educational Recommender Systems," IEEE Trans. Learning Technol., vol. 10, pp. 405-418, 2017.

[38] A. C. Rivera, M. Tapia-Leon, and S. LujanMora, "Recommendation Systems in Education: A Systematic Mapping Study," in Proceedings of the International Conference on Information Technology \& Systems (ICITS 2018), vol. 721. Á. Rocha, T. Guarda, Eds. Cham: Springer International Publishing, 2018, pp. 937-947.

[39] Y. Park and Y. Kim, "A Design and Development of micro-Learning Content in e-Learning System," International Journal on Advanced Science Engineering Information Technology, vol. 8, 2018.

[40] A. A. Kardan and O. R. Speily, "Smart Lifelong Learning System Based on Q-Learning," in 2010 Seventh International Conference on Information Technology: New Generations: IEEE, 2010,pp. 1086-1091.

[41] R. D. Araujo, R. G. Cattelan, and F. A. Dorca, "Towards an Adaptive and Ubiquitous Learning Architecture," in 2017 IEEE 17th International Conference on Advanced Learning Technologies (ICALT): IEEE, 2017, pp. 539-541.

[42] C. Na, Z. Xiuyuan, and L. Na, "Research on Network Personalized Learning Recommendation Algorithm Based on Knowledge Representation," in 2020 International Conference on Information Science, Parallel and Distributed Systems (ISPDS): IEEE, 2020, pp. 348-351.

[43] R. Raman and P. Nedungadi, "Performance improvements in schools with Adaptive Learning and Assessment," in 2010 4th International Conference on Distance Learning and Education: IEEE, 2010.

[44] C. G. Brinton, R. Rill, S. Ha, M. Chiang, R. Smith, and W. Ju, "Individualization for Education at Scale: MIIC Design and Preliminary Evaluation," IEEE Trans. Learning Technol., vol. 8, pp. 136-148, 2015.

[45] Z. Shou, X. Lu, Z. Wu, H. Yuan, H. Zhang, and J. Lai, "On Learning Path Planning Algorithm Based on Collaborative Analysis of Learning Behavior," IEEE Access, vol. 8, pp. 119863119879, 2020.

[46] D. Samia and A. Abdelkrim, "An adaptive educationnal hypermedia system integrating learning styles: Model and experiment," in International Conference on Education and e-Learning Innovations: IEEE, 2012, pp. 1-6.

[47] M. Marinov and I. Valova, "Application of planning techniques in knowledge-managed tutoring systems," in 2016 15th International Conference on Information Technology Based Higher Education and Training (ITHET): IEEE, 2016, pp. 1-5.

[48] L. Brown, R. Kerwin, and A. M. Howard, “Applying Behavioral Strategies for Student Engagement Using a Robotic Educational Agent," in 2013 IEEE International Conference on Systems, Man, and Cybernetics: IEEE, 2013, pp. 4360-4365.

[49] A. Zouhair, E. M. En-Naimi, B. Amami, H. Boukachour, P. Person, and C. Bertelle, "MultiAgent case-based reasoning and individualized follow-up of learner in remote learning," in 2011 International Conference on Multimedia Computing and Systems: IEEE, 2011, pp. 1-6.

[50] C. Troussas, A. Krouska, and M. Virvou, "NLPbased error analysis and dynamic motivation techniques in mobile learning," in 2019 10th International Conference on Information, Intelligence, Systems and Applications (IISA): IEEE, 2019, pp. 1-8.

[51] W. Cui, Z. Xue, and K.-P. Thai, "Performance Comparison of an AI-Based Adaptive Learning System in China," in 2018 Chinese Automation Congress (CAC): IEEE, 2018, pp. 3170-3175.

[52] W.-H. Kim and J.-H. Kim, "Individualized AI Tutor Based on Developmental Learning Networks," IEEE Access, vol. 8, pp. 27927-27937, 2020.

[53] Y. Lian, "An online adaptive tutoring system for design-centric courses," in 2011 IEEE International Symposium of Circuits and Systems (ISCAS): IEEE, 2011, pp. 1191-1194. 
[54] M. A. Hasan, N. F. M. Noor, S. S. B. A. Rahman, and M. M. Rahman, "The Transition From Intelligent to Affective Tutoring System: A Review and Open Issues," IEEE Access, vol. 8, pp. 204612-204638, 2020.

[55] Y. Huang, Y. Li, J. Shao, J. Ding, and J. Wang, "A clustering algorithm based on Bayesian network learning system," in Proceedings of 2011 International Conference on Computer Science and Network Technology: IEEE, 2011, pp. 557559.

[56] A. Zouhair, E. M. En-Naimi, B. Amami, H. Boukachour, P. Person, and C. Bertelle, "Intelligent tutoring systems founded on the multi-agent incremental dynamic case based reasoning," in 2012 Colloquium in Information Science and Technology: IEEE, 2012, pp. 74-79.

[57] C. Troussas, A. Krouska, and M. Virvou, "Adaptive e-learning interactions using dynamic clustering of learners' characteristics," in 2019 10th International Conference on Information, Intelligence, Systems and Applications (IISA): IEEE, 2019, pp. 1-7.

[58] R. Hare, Y. Tang, W. Cui, and J. Liang, "Optimize Student Learning via Random ForestBased Adaptive Narrative Game," in 2020 IEEE 16th International Conference on Automation Science and Engineering (CASE): IEEE, 2020, pp. 792-797.

[59] P. Nedungadi and R. Raman, "Effectiveness of adaptive learning with interactive animations and simulations," in 20103 rd International Conference on Advanced Computer Theory and Engineering(ICACTE): IEEE, 2010, V6-40-V6-44.

[60] A. Zouhair, E. M. En-Naimi, B. Amami, H. Boukachour, P. Person, and C. Bertelle, "Intelligent tutoring systems founded of incremental dynamic case based reasoning and multi-agent systems (ITS-IDCBR-MAS)," in 2013 International Conference on Advanced Logistics and Transport: IEEE, 2013, pp. 341-346.

[61] P. Nedungadi and M. S. Remya, "Incorporating forgetting in the Personalized, Clustered, Bayesian Knowledge Tracing (PC-BKT) model," in 2015 International Conference on Cognitive Computing and Information Processing(CCIP): IEEE, 2015, pp. 1-5.

[62] F. M. Felder and B. Soloman, "Index of Learning Styles," North Carolina State University, 1991.

[63] D. A. Kolb, Experiential learning: Experience as the source of learning and development. New Jersey-Hall, 1984.
[64] O. Bourkoukou, E. E. Bachari, and M. E. Adnani, "A Personalized E-Learning Based on Recommender System," IJLT, 2016.

[65] K. Almohammadi and H. Hagras, "An adaptive fuzzy logic based system for improved knowledge delivery within intelligent E-Learning platforms," in 2013 IEEE International Conference on Fuzzy Systems (FUZZ-IEEE): IEEE, 2013, pp. 1-8.

[66] A. A. Elghali, "New model of ALTENN implementation using Neural Network," in 2016 17th International Conference on Sciences and Techniques of Automatic Control and Computer Engineering (STA): IEEE, 2016, pp. 1-7.

[67] S. Lai, B. Sun, F. Wu, and R. Xiao, "Automatic Personality Identification Using Students' Online Learning Behavior," IEEE Trans. Learning Technol., vol. 13, pp. 26-37, 2020.

[68] R. M. Carro and V. Sanchez-Horreo, "The effect of personality and learning styles on individual and collaborative learning: Obtaining criteria for adaptation," in 2017 IEEE Global Engineering Education Conference (EDUCON): IEEE, 2017, pp. 1585-1590.

[69] Z. Papamitsiou and A. A. Economides, "The Effect of Personality Traits on Students' Performance during Computer-Based Testing: A Study of the Big Five Inventory with Temporal Learning Analytics," in 2014 IEEE 14th International Conference on Advanced Learning Technologies: IEEE, 2014, pp. 378-382.

[70] P. Sommerauer and O. Müller, "Augmented reality for teaching and learning - A literature review on theoretical and empirical foundations," European Conference on Information Systems, 2021. 